\title{
Spectral Line Shapes in Plasmas II
}

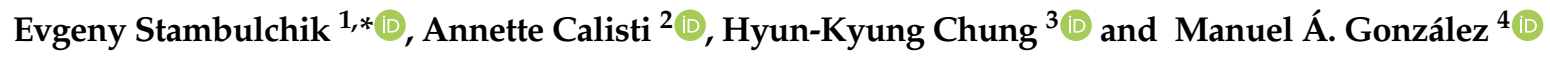 \\ 1 Faculty of Physics, Weizmann Institute of Science, Rehovot 7610001, Israel \\ 2 Aix Marseille Université, CNRS, PIIM UMR 7345, 13397 Marseille, France; annette.calisti@univ-amu.fr \\ 3 National Fusion Research Institute, Daejeon 34133, Korea; hchung.hedp@gmail.com \\ 4 Departamento de Física Aplicada, Universidad de Valladolid, 47011 Valladolid, Spain; \\ manuelgd@termo.uva.es \\ * Correspondence: Evgeny.Stambulchik@weizmann.ac.il; Tel.: +972-8-934-3610
}

Received: 28 January 2019; Accepted: 30 January 2019; Published: 6 February 2019

check for updates

\section{Foreword}

The Spectral Line Shapes in Plasmas (SLSP) code comparison workshop series [1] has gained a steady momentum, with four meetings organized thus far-in 2012, 2013, 2015, and 2017. A large number of diverse problems have been analyzed, advancing understanding of the phenomena involved and increasing accuracy of the models. Doubtlessly, this has significantly aided in improving theoretical aspects of line-shape analysis - one of the most important tools for diagnostics of both laboratory and space plasmas.

The first Special Issue of Atoms under this title was published in 2014 [2,3], covering selected topics from the first two workshops. With the hope of establishing tradition, we decided to arrange for the present Special Issue as a place for disseminating new results obtained in the course of the 3rd and 4th SLSP workshops.

\section{New Codes and Algorithms}

For a code comparison workshop, the appearance of new codes and algorithms is most welcome. One of the newcomers is ZEST by Gilleron and Pain [4], a fast code for simulating Zeeman-Stark line-shape functions, which debuted at SLSP4. The code is based on the "standard theory" of line broadening, but with the ion dynamics accounted according to the frequency-fluctuation model.

Lineshape data analysis is typically performed by varying a set of plasma parameters until the calculated spectrum fits best the experimental one. This straightforward approach soon becomes unmanageable in case of complex (multi-line, multi-species) spectra and/or a large number of free parameters. This is where genetic algorithms shine. Indeed, Mossé et al. [5] show how a genetic algorithm can be coupled with the PPP lineshape code, and use this method to analyze experimental spectra of Balmer- $\beta$ and He I 492.2-nm lines in a corona discharge. It is noteworthy to compare results of this study with multi-code analyses of the Balmer- $\beta$ and He I 492.2-nm lines done separately by Sheeba et al. in $[6,7]$, respectively. In the latter, it is also shown that accounting for line broadening by neutrals can be crucial for correct data interpretation.

Lineshape codes rarely calculate atomic data required for the line broadening themselves, relying instead on external databases or atomic codes. Atomic structure of $N$-electron atoms is often determined using the Hartree-Fock method, which typically involves solving a set of integro-differential equations. In this volume, Gomez et al. [8] describe how this can be done using a matrix method. 


\section{Hydrogen-Like Transitions}

Contrary to complex many-electron atoms, hydrogen or hydrogen-like ions are text-book examples of quantum systems for which availability of highly accurate atomic data is not an issue. Nevertheless, such systems do present unsolved-yet puzzles for lineshape calculations.

The asymmetry of hydrogen-like line profiles, observed at higher plasma densities, require going beyond the widely used dipole linear Stark effect approximation, which also implies considering quadrupole and higher-order multipole expansion terms. Demura [9] reviews the long, and at times twisted, history of the developments in this area. It is notorious that, in spite of all the efforts, there are still issues that are not viewed unanimously by the community.

The topic of discrete line merging and continuum lowering is of broad interest; it is also another area where applicability of the linear-Stark-effect approximation may be questioned. The comparative study of Alexiou et al. [10] shows how different models approach this problem.

The non-linear corrections are typically minor compared to the linewidths, which complicates the measurements. The new data of the He II Paschen- $\alpha$ width and shift, presented by Stollberg et al. [11], are compared to calculations, and the observed width-shift relation appears to be inconsistent with the theoretical predictions. It is expected that further efforts-both on the experimental and theoretical sides-will put an end to the controversy.

It is not uncommon to observe H-like transitions subject to both the plasma electric microfields and the macroscopic magnetic field; perhaps the most familiar cases are tokamak devices and atmospheres of white dwarfs. In a majority of the studies of lineshapes emitted from magnetized plasmas, the influence of the magnetic field on the trajectories of the plasma particles is neglected, however. Rosato et al. [12] illustrate the effect of the modified plasma-particle motion assuming an ideal one-component plasma.

\section{Isolated Lines}

Stark broadening of the isolated lines usually requires considering many contributing atomic states. By imposing an artificially simple atomic model (essentially, a two-level system), the calculation cases of the two last workshops reduced this complexity, allowing for a more straightforward comparison of various models at a deeper level. Such a comparison is presented by Sahal-Bréchot et al. [13].

Although the chemical composition of the universe is largely dominated by hydrogen and helium, it is the spectra of the rest of the Mendeleev's table species that provide us with abundant information on the stars and other space objects. However, the Stark broadening of only a minor part of all the lines observed has been calculated. Dimitrijević and Chougule [14] continue filling the gaps by providing Stark-broadening parameters of several Cr III transitions.

\section{Conclusions}

The topic of lineshape formation in plasma remains of high importance. It is a vibrant field, with many new developments published regularly, but also with some old puzzles that still remain to be solved. The results presented in this Special Issue represent only a part of the SLSP workshop outcomes, with more studies expected to be published elsewhere. The next, fifth workshop is scheduled for May 2019 in Vrdnik, Serbia [1].

Acknowledgments: The organizational and financial support from the International Atomic Energy Agency for conducting the SLSP workshops is highly appreciated.

Conflicts of Interest: The authors declare no conflict of interest. 


\section{References}

1. Spectral Line Shapes in Plasmas Workshops. Available online: http://plasma-gate.weizmann.ac.il/slsp/ (accessed on 28 January 2019).

2. Stambulchik, E.; Calisti, A.; Chung, H.K.; González, M.Á. Special Issue on Spectral Line Shapes in Plasmas. Atoms 2014, 2, 378-381. [CrossRef]

3. Stambulchik, E.; Calisti, A.; Chung, H.K.; González, M.Á. (Eds.) Spectral Line Shapes in Plasmas; MDPI: Basel, Switzerland, 2015; Printed Edition of the Special Issue Published in Atoms.

4. Gilleron, F.; Pain, J.C. ZEST: A fast code for simulating Zeeman-Stark line-shape functions. Atoms 2018, 6, 11. [CrossRef]

5. Mossé, C.; Génésio, P.; Bonifaci, N.; Calisti, A. A new procedure to determine the plasma parameters from a genetic algorithm coupled with the spectral line-shape code PPP. Atoms 2018, 6, 55. [CrossRef]

6. Sheeba, R.R.; Koubiti, M.; Bonifaci, N.; Gilleron, F.; Mossé, C.; Pain, J.C.; Rosato, J.; Stambulchik, E. H $\beta$ line in a corona helium plasma: A multi-code line shape comparison. Atoms 2018, 6, 29. [CrossRef]

7. Sheeba, R.R.; Koubiti, M.; Bonifaci, N.; Gilleron, F.; Pain, J.C.; Stambulchik, E. Broadening of the neutral helium 492-nm line in a corona discharge: Code comparisons and data fitting. Atoms 2018, 6, 19. [CrossRef]

8. Gomez, T.; Nagayama, T.; Fontes, C.; Kilcrease, D.; Hansen, S.; Montgomery, M.; Winget, D. Matrix methods for solving Hartree-Fock equations in atomic structure calculations and line broadening. Atoms 2018, 6, 22. [CrossRef]

9. Demura, A.V. Beyond the linear Stark effect: A retrospective. Atoms 2018, 6, 33. [CrossRef]

10. Alexiou, S.; Stambulchik, E.; Gomez, T.; Koubiti, M. The Fourth Workshop on Lineshape Code Comparison: Line merging. Atoms 2018, 6, 13. [CrossRef]

11. Stollberg, C.; Stambulchik, E.; Duan, B.; Gigosos, M.A.; Herrero, D.G.; Iglesias, C.A.; Mossé, C. Revisiting Stark width and shift of He II P $\alpha$. Atoms 2018, 6, 23. [CrossRef]

12. Rosato, J.; Ferri, S.; Stamm, R. Influence of helical trajectories of perturbers on Stark line shapes in magnetized plasmas. Atoms 2018, 6, 12. [CrossRef]

13. Sahal-Bréchot, S.; Stambulchik, E.; Dimitrijević, M.S.; Alexiou, S.; Duan, B.; Bonifaci, N. The Third and Fourth Workshops on Spectral Line Shapes in Plasma Code Comparison: Isolated lines. Atoms 2018, 6, 30. [CrossRef]

14. Dimitrijević, M.S.; Chougule, A. Stark broadening of Cr III spectral lines: DO white dwarfs. Atoms 2018, 6, 15. [CrossRef]

(C) 2019 by the authors. Licensee MDPI, Basel, Switzerland. This article is an open access article distributed under the terms and conditions of the Creative Commons Attribution (CC BY) license (http://creativecommons.org/licenses/by/4.0/). 